\title{
Review Article \\ Leukemia Inhibitory Factor: Roles in Embryo Implantation and in Nonhormonal Contraception
}

\author{
Naguib Salleh and Nelli Giribabu \\ Department of Physiology, Faculty of Medicine, University of Malaya, 50603 Kuala Lumpur, Malaysia \\ Correspondence should be addressed to Naguib Salleh; naguib.salleh@yahoo.com.my
}

Received 1 April 2014; Accepted 9 June 2014; Published 24 July 2014

Academic Editor: Víctor Sánchez-Margalet

Copyright (C) 2014 N. Salleh and N. Giribabu. This is an open access article distributed under the Creative Commons Attribution License, which permits unrestricted use, distribution, and reproduction in any medium, provided the original work is properly cited.

Leukaemia inhibitory factor (LIF) plays an indispensible role in embryo implantation. Aberrant LIF production is linked to implantation failure. LIF regulates multiple processes prior to and during implantation such as uterine transformation into a receptive state, decidualization, blastocyst growth and development, embryo-endometrial interaction, trophoblast invasion, and immune modulation. Due to its critical role, LIF has been a target for a nonhormonal contraception. In this review, we summarize up-to-date information on the role of LIF in implantation and its role in contraception.

\section{Introduction}

Leukemia inhibitory factor (LIF), a pleiotropic cytokine from interleukin- (IL-) 6 family, regulates various cellular functions via binding to membrane-bound LIF receptor (LIFR) and gp130 [1]. Currently, three spliced variants of LIF have been identified which include membrane-associated, diffusible, and truncated forms acting as paracrine factors in embryo implantation [2]. Binding of LIF to LIFR recruits gp130 to form high affinity functional receptor complex leading to activation of downstream signal transduction pathway such as signal transducer and activator of transcription (STAT) [3]. In addition to the membrane-bound receptor, a number of soluble forms of LIF receptor have been identified which are involved in either potentiating or dampening LIF activities. The soluble forms of LIFR and gp130 can function as antagonists that compete with membrane-bound receptor for the binding to LIF [4]. Meanwhile, suppressor of cytokine signaling 3 (SOCS3) can also inhibit LIF signaling and can act as a negative regulator for LIF action [5]. Following binding of LIF to LIFR, SOCS3 inhibits LIF action via JAK1STAT3 signaling pathway [6]. SOCS3 can also attenuate other signaling cascades which are induced upon LIF binding to LIFR and gp130 such as ERK-MAPK signaling pathway [7]. Few studies have demonstrated that LIF, gp130, and STAT are crucial for embryo implantation. Failure of blastocyst to implant has been reported in LIF gene knockout mice [8]. Meanwhile, mice with gp130 mutation and STAT-binding site deletion are also infertile indicating that gp130 and STAT are essential in regulating LIF action [3]. In species such as mice, uterine LIF displays biphasic expression pattern with the first peak appearing in the glands in preparation for uterine receptivity while the second peak appears in the stroma surrounding the implanting blastocyst at the time of attachment reaction [9]. In parallel, LIFR and gp130 are expressed in the luminal epithelia and stroma throughout the peri-implantation period [9] which further reinforce the critical role of LIF in embryo implantation.

Ovarian steroids are reported to play important role in regulating LIF, LIFR, and gp130 expressions in the uterus throughout the implantation window period. In mice, endometrial LIF secretion can be induced by nidatory estrogen at day 4 of pregnancy [10] while exogenous estrogen and progesterone administration to ovariectomised mice were able to increase gp130 expression in the uterine glands [11]. However, in humans, a report has indicated that luteal estrogen was not required to initiate the implantation process [12]. In hamsters, LIF secretion was induced by estrogen while the expression of LIFR and gp130 was induced by progesterone [12]. Currently, there is limited information with regard to regulations of LIF, LIFR, and gp130 expression in humans. An in vitro study using human endometrial stromal cell line 
indicated that concomitant administration of estrogen and progesterone was able to upregulate LIF receptor mRNA expression [13]. In humans, chorionic gonadotrophins (hCG) was also reported to upregulate LIF expression [14]. hCG and transforming growth factor- (TGF-) $\beta$ increase LIF secretion by the cultured endometrial epithelial cells derived from follicular and secretory phases of the menstrual cycle [15]. Meanwhile, male seminal fluid was also found to stimulate LIF secretion by human endometrial epithelial cells in vitro [16].

Several strands of clinical evidences indicated important role of LIF during human embryo implantation. A moderate to high LIF expression was detected during the proliferative and secretory phases of the menstrual cycle in normal fertile women with low expression observed in infertile women with implantation failure. However, no differences in endometrial expression of gp130 were noted between fertile and infertile women [17]. Further assessment of uterine luminal fluid indicated that endometrium of infertile women secretes significantly lesser amount of LIF and gp130 than normal fertile women [18] between luteal days (LH) 6 to 13 which coincides with implantation window period [19].

Evidences have shown that LIF is involved in the following events during implantation which include (i) endometrial transformation into a receptive state [2], (ii) embryoendometrial interaction [20], (iii) stromal decidualization [21], (iv) trophoblast invasion [22], (v) blastocyst growth and development [8], and (vi) uterine leukocyte infiltration [13]. LIF has also been found to play an important role in regulating synthesis of prostaglandins (PGs), an important mediator of implantation and decidualization [23]. This review summarizes the current knowledge on the role of LIF in embryo implantation which could be used to guide further research in this field. Additionally, potential application of LIF as a target for nonhormonal contraception was also discussed. Figure 1 summarizes the role of LIF in multiple steps during embryo implantation and placentation.

\section{LIF Role in Uterine Transformation into a Receptive State}

At the beginning of implantation window period in human, the expression of chicken ovalbumin upstream promoter transcription factor (COUP-TF) II, which is encoded by NR2F2 gene [24] was increased in uterine stroma under the influence of progesterone [25]. This increase will result in suppression of uterine luminal epithelial cell proliferation via inhibition on estrogen receptor- (ER-) $\alpha$ activity [26]. Meanwhile, another endometrial transcription factor, Hand2, which was upregulated by progesterone also inhibits fibroblast growth factor- (FGF-) induced epithelial cell proliferation via downregulating ER- $\alpha$ expression and ERK1/2 signaling pathway in uterine luminal epithelia [27]. The role of LIF in the inhibition of epithelial proliferation at the onset of uterine receptivity period remains elusive. During uterine, receptivity, several changes in protein expression have been reported to occur in the uterine luminal epithelia which include increased synthesis of epithelial growth factor (EGF), for example, heparin-binding epidermal growth factor (HBEGF) and its receptors, ErbB1 and ErbB2 [28]. In addition, increased expression of cytokines [18, 29] and intercellular adhesion molecules such as ICAM and fibrinogen- $\gamma$ (FGG) has also been documented during this period [30].

LIF prepares the endometrium for embryo implantation. Several reports have indicated that in mice, peak expression of LIF occurs in the glands at the time of ovulation and prior to the onset of implantation [31, 32]. Epithelial-derived LIF was reported to act as autocrine regulator in the preparation of endometrium for implantation [8]. Female mice lacking of LIF gene suffered from implantation failure [32]. Meanwhile in humans, LIF expression in the endometrium was restricted to the glands, which was the highest during midluteal phase of the cycle [33]. In fertile women, LIF was also detected in uterine luminal fluid during the luteal phase of the menstrual cycle [14] and at the expected time of implantation [34]. In parallel, expression of LIFR- $\beta$ was reported to be the highest in the luminal epithelia during secretory phases of the menstrual cycle while expression of gpl30 was found both in the luminal and glandular epithelia throughout menstrual cycle phases [20].

During receptivity period, LIF either binds directly to LIFR which is expressed on the blastocyst [35] or endometrial surfaces, in which the latter participates in autoregulation of LIF secretion [4]. LIF affects synthesis of growth factors in the endometrial epithelia. In LIF-deficient female mice, EGF-like growth factors such as amphiregulin (Ar), heparin binding epidermal growth factor (HB-EGF), and epiregulin (Ereg) were not expressed at the site of blastocyst apposition [36], although expressions of EGF receptors were not affected [36]. The dependency of Ar on LIF was evident from lack of expression of this growth factor in uterine luminal epithelia following administration of inhibitor to LIF (hLIF-05) [37]. LIF was also required to induce expression of implantation genes including Msx-1 and Wnt-4 [38]. Despite of these effects, direct role of LIF in regulating expression of adhesion molecules such as L-selectins, E-cadherins [39] and tight junction proteins, for example, claudin and occludin [40] which are expressed in the receptive endometrium remains elusive.

\section{LIF Role in Decidualization}

During the luteal phase of menstrual cycle and diestrus stage of oestrous cycle, stromal cells proliferate and differentiate into decidual cells which then produced various factors that help to prepare endometrium for blastocyst adhesion and subsequently trophoblast invasion. CCAAT/enhancerbinding protein $\beta(\mathrm{C} / \mathrm{EBP} \beta)$ is a transcription factor that has been identified as a regulator of uterine stromal cell proliferation and differentiation in mice [41] and humans [21]. $\mathrm{C} / \mathrm{EBP} \beta$ controls proliferation of primary human endometrial stromal cells (HESCs) in vitro by regulating expression of several key cell cycle-regulatory factors [42]. C/EBP $\beta$ also increases the response of HESCs to estrogen, progesterone, and cyclic AMP (cAMP) and regulates interleukin- (IL-) 11 receptor and its downstream STAT3 transcription factor 


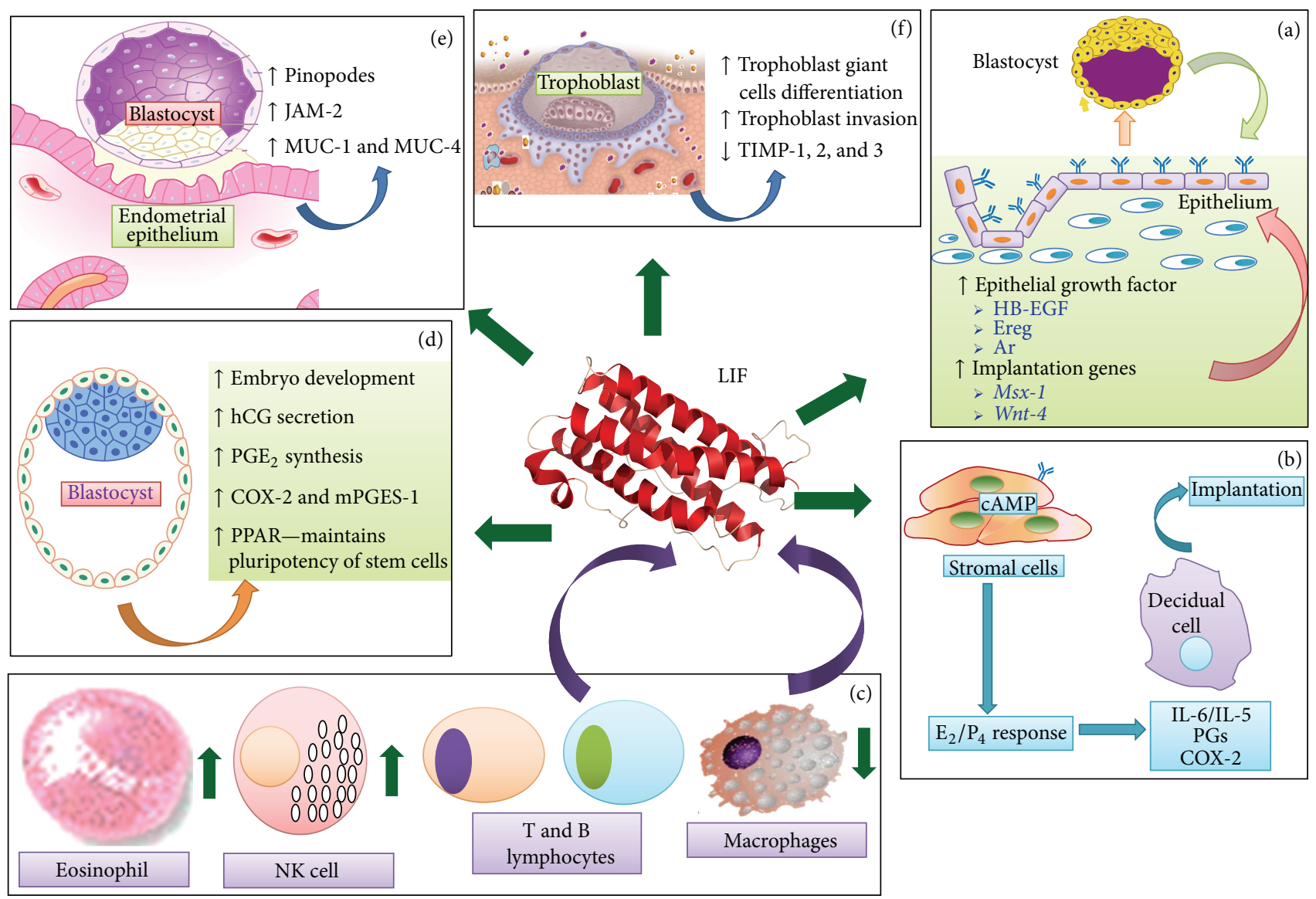

FIGURE 1: Summary of the known roles of LIF in embryo implantation. LIF increases the expression of EGF and implantation genes in receptive endometrium. LIF produced by endometrium and blastocyst regulates growth and development of the embryo. Meanwhile, LIF stimulates stromal decidualization by increasing the production of cytokines and prostaglandins. LIF is also involved in enhancing embryo-endometrial interaction through pinopodes and adhesion molecules. LIF stimulates trophoblast cells differentiation and increase trophoblast capability to invade the uterine stroma. Finally, LIF is involved in recruitment of specific cohort of leucocytes which participates in inflammatory response during implantation. LIF: leukemia inhibitory factor, HB-EGF: heparin binding-epidermal growth factor, Ereg: epiregulin, Ar: amphiregulin, E2: estrogen, P4: progesterone, IL: interleukins, PGs: prostaglandins, COX: cyclo-oxygenase, NK: natural killer, PPAR: peroxisome proliferator-activated receptor, PGE2: prostaglandins E2, hCG: human chorionic gonadotrophin, MUC: mucin, JAM: junctional adhesion molecules.

expression [21]. Female mice lacking $\mathrm{C} / \mathrm{EBP} \beta$ gene are infertile with their uteri irresponsive towards deciduogenic stimuli while proliferation and differentiation of stromal cells were also impaired [43].

LIF plays important role in decidualization. Failure of stromal cells to differentiate into primary decidual cells has been reported in LIF-deficient mice [44]. LIF also enhances estrogen and progesterone-induced decidualization in HESCs via STAT3 phosphorylation [13]. Meanwhile, LIF was also found to upregulate the secretion of IL-6 and IL-15 from decidualized HESCs in vitro [13]. During decidualization, SOCS3 protein is stimulated in response to cytokineinduced STAT3 phosphorylation which acts as a negativefeedback inhibitor to hinder LIFR activity [45]. LIF was reported to indirectly stimulate the synthesis of PGs which is an important mediator of decidualization via IL-1 [46] and is required for cyclooxygenase-2 (COX-2) expression, in which the latter is a rate-limiting enzyme in the PGs synthesis [36].
Female mice lacking LIF gene suffered from implantation failure due to impaired PGs synthesis [36].

\section{LIF Role in Leukocyte Recruitment during Implantation}

In early pregnancy, infiltration of immune cells such as dendritic cells (DC), macrophages, $\mathrm{T}$ and $\mathrm{B}$ lymphocytes, natural killer (NK) cells [47], and neutrophils and eosinophils [48] into the endometrium was initially stimulated by factors in the seminal fluid [49] and later by the implanting blastocyst [50]. DCs are involved in immune tolerance, tissue remodeling, angiogenesis, and development of $\mathrm{T}$ regulatory (Treg) cells [51]. In humans, primary unexplained infertility was found to be associated with reduced expression of Treg in the endometrial tissue [52]. Macrophages participate in the progression of inflammation, counteract nitric oxide synthesis, tissue remodeling, angiogenesis, and immune tolerance 
towards the implanting blastocyst [53]. Meanwhile, T cells produced type- 1 and type- 2 cytokines which are involved in proinflammatory and anti-inflammatory responses in which changes in their ratio would determine the success of implantation [54]. In the late secretory phase and in early pregnancy, percentage of endometrium/decidual NK cells increases rapidly reaching up to $70 \%$ of the total uterine leukocyte population [55]. However, following implantation, endometrial NK cells differentiate into decidual NK cells, which begin to secrete cytokines (TNF- $\alpha$, IL-10, GM-CSF, IL$1 \beta$, TGF- $\beta 1$, CSF-1, LIF, and IFN- $\gamma$ ), growth factors, angiogenic factors as well as being involved in tissue remodelling, trophoblast migration, and decidualization [56].

LIF was reported to play important role in the regulation of immune response in the uterus in early pregnancy. LIF affects uterine leukocyte subpopulation and recruits specific cohort of leucocytes to the site of implantation [57]. LIF mRNA is expressed in decidual leucocytes itself [58]. LIFdeficient mice were found to have increased number of uterine macrophages although the number of NK cells and eosinophils [48] was reduced. Macrophage-derived LIF facilitates development of implantation-receptive endometrium by modulating the surface glycan structure of epithelial cells [59] as well as regulating the expression of fucosyltransferase enzyme in the uterine epithelia which is involved in the synthesis of embryo adhesive fucosylated glycoconjugates during the period of inflammatory response towards insemination [60]. A recent study reported that intrauterine administration of peripheral blood mononuclear cells (PBMCs) in mice helped to improve endometrial receptivity as evidence from a high pregnancy rate associated with increased endometrial LIF and vascular endothelial growth factor (VEGF) expressions [61]. PBMCs were found to produce cytokines and angiogenic factors necessary for implantation [56]. A study in humans has indicated that intrauterine infusion of PBMCs could help to improve the clinical pregnancy rate in patients with repeated implantation failure during in vitro fertilization-embryo transfer (IVF-ET) procedure [62], indicating that leucocytes play important role in ensuring the success of human embryo implantation.

\section{LIF Role in Blastocyst Growth and Development}

Following fertilization, embryo divides from 2- to 4-cell stages and subsequently 8-cell stage, in which the latter formed a morula which then develops into blastocyst that hatches upon entering the uterine cavity [63]. Blastocyst is then brought closer to the uterine wall by a force generated from fluid reabsorption in the uterine glands [64]. During blastocyst development, pluripotent inner cells are prepared for specific differentiation while outer trophectoderm cells interact with uterine epithelium in preparation for trophoblast invasion [65]. Mouse blastocysts were reported to express LIF mRNA transcript [66] which helps to increase rate of preimplantation embryo development $[67,68]$. Meanwhile, mouse [69], rabbit [70] and human [71] blastocysts expressed LIFR and gp130 where the latter promotes preimplantation human embryo development in culture [72]. A combined administration of insulin-like growth factor(IGF-) I, $\beta$-fibroblast growth factor (FGF), transforming growth factor- (TGF-) $\beta 1$, granulocyte-monocyte colony stimulating factor (GM-CSF), and LIF have been reported to accelerate blastocyst development in vitro, especially changes from expanded to hatched blastocyst stages [73].

Leptin, a hormone linked to fertility acted via LIF to cause increased proportion of hatched blastocysts while causing decreased rate of embryo cell apoptosis in vitro via STAT3 signaling pathway [74]. LIF was found to affect hCG secretion by the trophoblast cells in vitro [75]. LIF was also reported to induce prostaglandin $\mathrm{E}\left(\mathrm{PGE}_{2}\right)$ production by human trophoblast cell line via stimulating COX-2 and microsomal PGE synthase-1 (mPGES-1) enzymes expression that are involved in PGE synthesis [23]. Meanwhile, LIF maintains pluripotency of mouse embryonic stem cells in culture via stimulating peroxisome proliferator-activated receptors (PPARs), a nuclear receptor transcription factors that regulates LIF-induced growth and self-renewal via tyrosine kinase 2-STAT3 signaling pathway [76].

\section{Role of LIF in Embryo-Endometrial Interaction}

During apposition phase of implantation, blastocyst initiates loose physical contact with the receptive endometrium which occurs prior to firm adhesion onto the endometrial surface. Mucin-1 (MUC-1), a glycocalyx which is expressed at the apical membrane of luminal epithelia, prevents firm blastocyst attachment [39]. At the site of trophoblast invasion, MUC1 expression was markedly reduced [77]. Lack or aberrant MUC-1 production was reported to be one of the reasons of ectopic pregnancy [78]. MUC-1 provides scaffold for Lselectin ligand, which binds L-selectin on the blastocyst surface, allowing loose physical contact between blastocyst and endometrium as well as facilitating blastocyst rolling over the endometrial surface [79]. Meanwhile, L-selectin ligand, which is expressed on the pinopode (uterodomes) surface [80], also helps in blastocyst rolling. Finally, increased expression of other adhesion molecules such as $\alpha \mathrm{v} \beta 3$ integrin [81], trophinins [82], junctional adhesion molecule (JAM) [83], and HB-EGF/errB4 complex [84] resulted in the blastocyst movement to come to a standstill which allows the blastocysts to firmly attach onto the endometrial surface prior to uterine invasion.

LIF plays indispensable role in initiating embryoendometrial interaction. In mice lacking of LIF, absence of pinopodes was observed [44]. Meanwhile, human endometrium with uterodomes at different stages of development had both luminal and glandular epithelia expressing high levels of LIF and LIFR in the luteal days 6 through to 9 [85]. A study has shown that expression of LIFR and gp130 in the endometrium of fertile women positively correlated with pinopode formation, while the opposite was observed in women with unexplained infertility. A reduced level of LIF has been reported in hydrosalpinx [86] but not in recurrent 
pregnancy loss [87]. LIF, involving STAT3 phosphorylation, was reported to induce the expression of JAM-2 adhesion molecule which mediates the interaction between hatched blastocyst and receptive endometrium between days 3 and 4 of pregnancy in mice [83]. Although LIF effect on the expression of other adhesion molecules remains elusive, synchronous reduction in the levels of LIF, integrin $\beta 3$, and MUC-1 was observed in the uterus of patients with hydrosalpinx [86], suggesting that the expression of these molecules was interdependent. Meanwhile, limited observation indicated that LIF affects expression of genes that encode antiadhesive mucins, MUC-1 and MUC-4 [60]. Other than these findings, LIF involvement in facilitating embryoendometrial interactions remains to be fully elucidated.

\section{Role of LIF in Trophoblast Invasion}

Trophoblast giant cells, the first cell lineage derived from trophoblast stem cells [88], have the ability to invade into the decidua to initiate the implantation reaction [89]. As it moves towards the uterine compartment, trophoblast cells are confronted by various extracellular matrix (ECM) proteins and basement membranes such as collagen, fibronectin, laminin, vitronectin, trophin, and tastin which are able to bind to integrins on the trophoblast surface. These molecules help in controlling adhesion, migration, differentiation, and spreading of the trophoblast cells [90]. Invasion involves degradation of extracellular matrix (ECM) elements in the direction of migration which requires involvement of protease enzymes, such as matrix metalloproteinases (MMPs) 2, 9 , and 14 [22] and is controlled by tissue inhibitor of metalloproteinases (TIMPs), for example, TIMPs 1, 2, and 3 [91].

LIF plays an important role in trophoblast invasion. LIF stimulates differentiation of trophoblast giant cells via JAK1-STAT3 pathway [6]. Meanwhile, soluble LIF provides extracellular signal that stimulates trophoblast invasion via STAT3 activation [92, 93]. LIF induces trophoblast cell proliferation via stimulating cell transition into $G(2) / M$ phase of the cell cycle and activates both STAT3 and ERK1/2 signaling cascades [94]. Recently, LIF has been shown to increase invasiveness of human trophoblast cell line (HTR8/SVneo cells) in vitro via STAT1 and STAT3 activation [95] as well as increase invasiveness of extravillous trophoblast cells via stimulating adhesion to the extracellular matrix elements including fibronectin, vitronectin, and laminin [96]. On the other hand, LIF was also reported to downregulate the expression of genes that encode TIMP1, TIMP2, and TIMP3 [95], therefore helping to reduce the expression of enzymes that are involved in potentiating trophoblast invasion. LIF was also reported to decrease the expression of integrin $\beta_{4}$ mRNA in the trophoblast cells which promotes trophoblast invasion [96].

\section{LIF as a Target for Nonhormonal Contraception}

A study in humans indicated that low concentration of LIF in the maternal plasma was associated with increased risks of early pregnancy loss during embryo transfer [97], which points towards critical role of LIF in ensuring the success of embryo implantation. However, despite of this report, administration of recombinant LIF during assisted reproductive techniques (ART) has revealed no improvement in implantation rates in women with recurrent unexplained implantation failure [98]. Meanwhile, LIF has been identified as a potential target in the development of nonhormonal birth control vaccine. A study in mice indicated that intraperitoneal injection of anti-LIF antibody inhibited embryo implantation [99] while immunization of female mice with LIF or LIFR peptide vaccines induced long-lasting antibody development which could block fertility [100]. A preliminary study in rhesus monkey indicated that administration of monoclonal antiLIF antibody could prevent embryo implantation [101].

In mice, intraperitoneal administration of LIF antagonist (LA) alone or conjugated to polyethylene glycol (PEGLA) between days 2.5 and 3.5 of pregnancy resulted in implantation failure which demonstrates that this compound could effectively be used as a nonhormonal contraceptive agent that targets LIF signaling in the endometrium [102]. Recently, vaginal administration of PEGLA in mice has been proven to be effective in inhibiting embryo implantation [103] while systemic administration of this compound to cynomolgus monkey reduced endometrial STAT3 phosphorylation, inhibited LIF-induced expression of cochlin, insulinlike growth factor-binding protein- (IGF-BP-) 3, vascular endothelial growth factor- (VEGF-) A, and COX-2 enzyme which are essential for embryo implantation [104]. While most works related to the use of LIF antagonist and PEGLA as nonhormonal contraceptive agents were preliminary and were limited to animal studies, a study using human tissue has been recently performed in vitro by Lalitkumar et al. [105]. In this study, the effect on human embryo attachment rate, embryo quality, and blastocyst expression of cell survival factor (Akt) and caspase-3 following exposure to endometrial tissue collected at luteal day $4(\mathrm{LH}+4)$ treated with PEGLA were determined. The findings indicated that in tissues treated with PEGLA, embryo attachment rate was reduced with embryonic LIF triggered apoptosis being inhibited. Meanwhile, endometrial LIF expression was also downregulated which was associated with the reduction in blastocyst survival rate and the increase in caspase- 3 expression in the blastocyst. Currently, no clinical trials have been conducted in humans to assess effectiveness of LA or PEGLA as nonhormonal contraceptive agents. Table 1 summarizes the studies performed using various models to investigate the effectiveness of LA or PEGLA as potential nonhormonal contraception agents.

\section{Perspective}

LIF is undoubtedly important in embryo implantation in rodents, primates, and humans. LIF has been shown to mediate multiple processes of embryo implantation ranging from blastocyst growth and development, uterine preparation for implantation, decidualization, uterine inflammatory responses towards the implanting embryos, embryoendometrial interaction, and trophoblast invasion. In view 


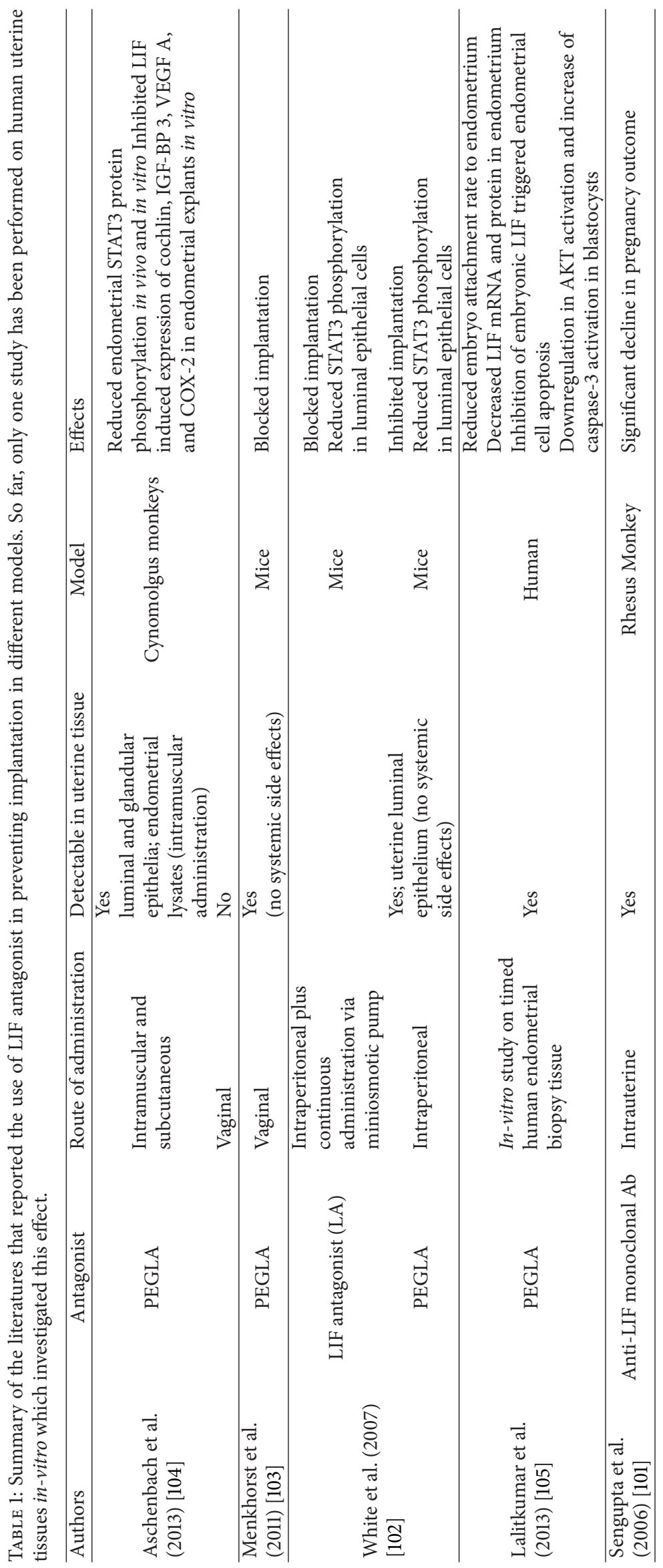


of these documented roles, LIF has been proposed as a potential target for nonhormonal contraception. While most information with regard to the mechanisms underlying LIF actions in uterus during implantation period was obtained mostly from studies involving rodents and endometrial cell lines, more works are needed in humans to elicit its role in blastocyst implantation.

\section{Conflict of Interests}

The authors have nothing to disclose.

\section{Acknowledgment}

Publication of this paper was supported by UMRG Grant (RG404/12HTM), University of Malaya, Kuala Lumpur, Malaysia.

\section{References}

[1] M.-E. Mathieu, C. Saucourt, V. Mournetas et al., "LIF-dependent signaling: new pieces in the lego," Stem Cell Reviews and Reports, vol. 8, no. 1, pp. 1-15, 2012.

[2] L. Aghajanova, "Leukemia inhibitory factor and human embryo implantation," Annals of the New York Academy of Sciences, vol. 1034, no. 1, pp. 176-183, 2004.

[3] X. Sun, A. Bartos, J. A. Whitsett, and S. K. Dey, "Uterine deletion of Gp130 or Stat3 shows implantation failure with increased estrogenic responses," Molecular Endocrinology, vol. 27, no. 9, pp. 1492-1501, 2013.

[4] S. Metz, G. Naeth, P. C. Heinrich, and G. Müller-Newen, "Novel inhibitors for murine and human leukemia inhibitory factor based on fused soluble receptors," Journal of Biological Chemistry, vol. 283, no. 10, pp. 5985-5995, 2008.

[5] Y. Takahashi, N. Carpino, J. C. Cross, M. Torres, E. Parganas, and J. N. Ihle, "SOCS3: an essential regulator of LIF receptor signaling in trophoblast giant cell differentiation," EMBO Journal, vol. 22, no. 3, pp. 372-384, 2003.

[6] Y. Takahashi, M. Takahashi, N. Carpino et al., "Leukemia inhibitory factor regulates trophoblast giant cell differentiation via janus kinase 1-signal transducer and activator of transcription 3-suppressor of cytokine signaling 3 pathway," Molecular Endocrinology, vol. 22, no. 7, pp. 1673-1681, 2008.

[7] K. Boyle and L. Robb, "The role of SOCS3 in modulating leukaemia inhibitory factor signalling during murine placental development," Journal of Reproductive Immunology, vol. 77, no. 1, pp. 1-6, 2008.

[8] C. L. Stewart, "Leukaemia inhibitory factor and the regulation of pre-implantation development of the mammalian embryo," Molecular Reproduction and Development, vol. 39, no. 2, pp. 233-238, 1994.

[9] H. Song and H. Lim, "Evidence for heterodimeric association of leukemia inhibitory factor (LIF) receptor and gp130 in the mouse uterus for LIF signaling during blastocyst implantation," Reproduction, vol. 131, no. 2, pp. 341-349, 2006.

[10] J. R. A. Sherwin, T. C. Freeman, R. J. Stephens et al., "Identification of genes regulated by leukemia-inhibitory factor in the mouse uterus at the time of implantation," Molecular Endocrinology, vol. 18, no. 9, pp. 2185-2195, 2004.
[11] H. Ni, N. Ding, M. J. K. Harper, and Z. Yang, "Expression of leukemia inhibitory factor receptor and gp130 in mouse uterus during early pregnancy," Molecular Reproduction and Development, vol. 63, no. 2, pp. 143-150, 2002.

[12] T. Ding, H. Song, X. Wang, A. Khatua, and B. C. Paria, "Leukemia inhibitory factor ligand-receptor signaling is important for uterine receptivity and implantation in golden hamsters (Mesocricetus auratus)," Reproduction, vol. 135, no. 1, pp. 41-53, 2008.

[13] L. L. Shuya, E. M. Menkhorst, J. Yap, P. Li, N. Lane, and E. Dimitriadis, "Leukemia inhibitory factor enhances endometrial stromal cell decidualization in humans and mice," PLoS ONE, vol. 6, no. 9, Article ID e25288, 2011.

[14] P. Licht, A. Lösch, R. Dittrich, J. Neuwinger, E. Siebzehnrübl, and L. Wildt, "Novel insights into human endometrial paracrinology and embryo-maternal communication by intrauterine microdialysis," Human Reproduction Update, vol. 4, no. 5, pp. 532-538, 1998.

[15] S. P. d'Hauterive, C. Charlet-Renard, S. Berndt et al., "Human chorionic gonadotropin and growth factors at the embryonicendometrial interface control leukemia inhibitory factor (LIF) and interleukin 6 (IL-6) secretion by human endometrial epithelium," Human Reproduction, vol. 19, no. 11, pp. 2633-2643, 2004.

[16] S. Gutsche, M. von Wolff, T. Strowitzki, and C. J. Thaler, "Seminal plasma induces mRNA expression of IL- $1 \beta$, IL- 6 and LIF in endometrial epithelial cells in vitro," Molecular Human Reproduction, vol. 9, no. 12, pp. 785-791, 2003.

[17] M. Wu, Y. Yin, M. Zhao, L. Hu, and Q. Chen, “The low expression of leukemia inhibitory factor in endometrium: possible relevant to unexplained infertility with multiple implantation failures," Cytokine, vol. 62, no. 2, pp. 334-339, 2013.

[18] M. A. Tawfeek, M. A. Eid, A. M. Hasan, M. Mostafa, and H. A. El-Serogy, "Assessment of leukemia inhibitory factor and glycoprotein 130 expression in endometrium and uterine flushing: a possible diagnostic tool for impaired fertility," BMC Women's Health, vol. 12, article 10, 2012.

[19] J. R. A. Sherwin, S. K. Smith, A. Wilson, and A. M. Sharkey, "Soluble gp130 is up-regulated in the implantation window and shows altered secretion in patients with primary unexplained infertility," Journal of Clinical Endocrinology and Metabolism, vol. 87, no. 8, pp. 3953-3960, 2002.

[20] E. B. Cullinan, S. J. Abbondanzo, P. S. Anderson, J. W. Pollard, B. A. Lessey, and C. L. Stewart, "Leukemia inhibitory factor (LIF) and LIF receptor expression in human endometrium suggests a potential autocrine/paracrine function in regulating embryo implantation," Proceedings of the National Academy of Sciences of the United States of America, vol. 93, no. 7, pp. 3115-3120, 1996.

[21] W. Wang, R. N. Taylor, I. C. Bagchi, and M. K. Bagchi, "Regulation of human endometrial stromal proliferation and differentiation by C/EBP $\beta$ involves cyclin E-cdk2 and STAT3," Molecular Endocrinology, vol. 26, no. 12, pp. 2016-2030, 2012.

[22] E. Staun-Ram and E. Shalev, "Human trophoblast function during the implantation process," Reproductive Biology and Endocrinology, vol. 3, no. 1, article 56, 2005.

[23] H. Horita, E. Kuroda, T. Hachisuga, M. Kashimura, and U. Yamashita, "Induction of prostaglandin E2 production by leukemia inhibitory factor promotes migration of first trimester extravillous trophoblast cell line, HTR-8/SVneo," Human Reproduction, vol. 22, no. 7, pp. 1801-1809, 2007. 
[24] Y. Qiu, V. Krishnan, Z. Zeng et al., "Isolation, characterization, and chromosomal localization of mouse and human COUP-TF I and II genes," Genomics, vol. 29, no. 1, pp. 240-246, 1995.

[25] I. Kurihara, D. Lee, F. G. Petit et al., "COUP-TFII mediates progesterone regulation of uterine implantation by controlling ER activity.", PLoS genetics, vol. 3, no. 6, article e102, 2007.

[26] D. Lee, I. Kurihara, J. Jeong et al., "Suppression of ER $\alpha$ activity by COUP-TFII is essential for successful implantation and decidualization," Molecular Endocrinology, vol. 24, no. 5, pp. 930-940, 2010.

[27] Q. Li, A. Kannan, F. J. DeMayo et al., “The antiproliferative action of progesterone in uterine epithelium is mediated by hand2," Science, vol. 331, no. 6019, pp. 912-916, 2011.

[28] X. Wang, H. Wang, H. Matsumoto, S. K. Roy, S. K. Das, and B. C. Paria, "Dual source and target of heparin-binding EGF-like growth factor during the onset of implantation in the hamster," Development, vol. 129, no. 17, pp. 4125-4134, 2002.

[29] N. Noda, H. Minoura, R. Nishiura et al., "Expression of Tenascin-C in stromal cells of the murine uterus during early pregnancy: Induction by interleukin- $1 \alpha$, prostaglandin E2, and prostaglandin $\mathrm{F}(2 \alpha)$," Biology of Reproduction, vol. 63, no. 6, pp. 1713-1720, 2000.

[30] L. Lecce, Y. Kaneko, R. J. Madawala, and C. R. Murphy, "ICAM1 and fibrinogen- $\gamma$ are increased in uterine epithelial cells at the time of implantation in rats," Molecular Reproduction and Development, vol. 78, no. 5, pp. 318-327, 2011.

[31] H. Bhatt, L. J. Brunet, and C. L. Stewart, "Uterine expression of leukemia inhibitory factor coincides with the onset of blastocyst implantation," Proceedings of the National Academy of Sciences of the United States of America, vol. 88, no. 24, pp. 11408-11412, 1991.

[32] C. L. Stewart, P. Kaspar, L. J. Brunet et al., "Blastocyst implantation depends on maternal expression of leukaemia inhibitory factor," Nature, vol. 359, no. 6390, pp. 76-79, 1992.

[33] D.-B. Chen, R. Hilsenrath, Z. Yang et al., "Leukaemia inhibitory factor in human endometrium during the menstrual cycle: cellular origin and action on production of glandular epithelial cell prostaglandin in vitro," Human Reproduction, vol. 10, no. 4, pp. 911-918, 1995.

[34] S. M. Laird, E. M. Tuckerman, C. F. Dalton, B. C. Dunphy, T. C. Li, and X. Zhang, "The production of leukaemia inhibitory factor by human endometrium: presence in uterine flushings and production by cells in culture," Human Reproduction, vol. 12, no. 3, pp. 569-574, 1997.

[35] D. S. Charnock-Jones, A. M. Sharkey, P. Fenwick, and S. K. Smith, "Leukaemia inhibitory factor mRNA concentration peaks in human endometrium at the time of implantation and the blastocyst contains mRNA for the receptor at this time," Journal of Reproduction and Fertility, vol. 101, no. 2, pp. 421-426, 1994.

[36] H. Song, H. Lim, S. K. Das, B. C. Paria, and S. K. Dey, "Dysregulation of EGF family of growth factors and COX-2 in the uterus during the preattachment and attachment reactions of the blastocyst with the luminal epithelium correlates with implantation failure in LIF-deficient mice," Molecular Endocrinology, vol. 14, no. 8, pp. 1147-1161, 2000.

[37] L. Mohamet, J. K. Heath, and S. J. Kimber, "Determining the LIF-sensitive period for implantation using a LIF-receptor antagonist," Reproduction, vol. 138, no. 5, pp. 827-836, 2009.

[38] T. Daikoku, H. Song, Y. Guo et al., "Uterine Msx-1 and Wnt4 signaling becomes aberrant in mice with the loss of leukemia inhibitory factor or Hoxa-10: evidence for a novel cytokine-homeobox-Wnt signaling in implantation," Molecular Endocrinology, vol. 18, no. 5, pp. 1238-1250, 2004.

[39] A. Sharma and P. Kumar, "Understanding implantation window, a crucial phenomenon," Journal of Human Reproductive Sciences, vol. 5, no. 1, pp. 2-6, 2012.

[40] M. C. Satterfield, K. A. Dunlap, K. Hayashi, R. C. Burghardt, T. E. Spencer, and F. W. Bazer, "Tight and adherens junctions in the ovine uterus: differential regulation by pregnancy and progesterone," Endocrinology, vol. 148, no. 8, pp. 3922-3931, 2007.

[41] S. R. Mantena, A. Kannan, Y. Cheon et al., " $C / E B P \beta$ is a critical mediator of steroid hormone-regulated cell proliferation and differentiation in the uterine epithelium and stroma," Proceedings of the National Academy of Sciences of the United States of America, vol. 103, no. 6, pp. 1870-1875, 2006.

[42] W. Wang, Q. Li, I. C. Bagchi, and M. K. Bagchi, "The CCAAT/ enhancer binding protein $\beta$ is a critical regulator of steroidinduced mitotic expansion of uterine stromal cells during decidualization," Endocrinology, vol. 151, no. 8, pp. 3929-3940, 2010.

[43] M. K. Bagchi, S. R. Mantena, A. Kannan, and I. C. Bagchi, "Control of uterine cell proliferation and differentiation by $\mathrm{C} / \mathrm{EBP} \beta$ : functional implications for establishment of early pregnancy," Cell Cycle, vol. 5, no. 9, pp. 922-925, 2006.

[44] A. A. Fouladi-Nashta, C. J. P. Jones, N. Nijjar et al., "Characterization of the uterine phenotype during the peri-implantation period for LIF-null, MF1 strain mice," Developmental Biology, vol. 281, no. 1, pp. 1-21, 2005.

[45] E. Dimitriadis, C. Stoikos, Y. Tan, and L. A. Salamonsen, "Interleukin 11 signaling components signal transducer and activator of transcription 3 (STAT3) and suppressor of cytokine signaling 3 (SOCS3) regulate human endometrial stromal cell differentiation," Endocrinology, vol. 147, no. 8, pp. 3809-3817, 2006.

[46] A. A. Fouladi-Nashta, L. Mohamet, J. J. Heath, and S. S. Kimber, "Interleukin 1 signaling is regulated by leukemia inhibitory factor (LIF) and is aberrant in Lif-/- mouse uterus," Biology of Reproduction, vol. 79, no. 1, pp. 142-153, 2008.

[47] J. Y. Lee, M. Lee, and S. K. Lee, "Role of endometrial immune cells in implantation," Clinical and Experimental Reproductive Medicine, vol. 38, no. 3, pp. 119-125, 2011.

[48] G. Schofield and S. J. Kimber, "Leukocyte subpopulations in the uteri of leukemia inhibitory factor knockout mice during early pregnancy," Biology of Reproduction, vol. 72, no. 4, pp. 872-878, 2005.

[49] S. A. Robertson, "Seminal fluid signaling in the female reproductive tract: lessons from rodents and pigs," Journal of Animal Science, vol. 85, supplement, no. 13, pp. E36-E44, 2007.

[50] R. Grümmer and E. Winterhager, "Blastocyst-mediated induction of endometrial connexins: an inflammatory response?" Journal of Reproductive Immunology, vol. 90, no. 1, pp. 9-13, 2011.

[51] V. Plaks, T. Birnberg, T. Berkutzki et al., "Uterine DCs are crucial for decidua formation during embryo implantation in mice," The Journal of Clinical Investigation, vol. 118, no. 12, pp. 3954-3965, 2008.

[52] M. J. Jasper, K. P. Tremellen, and S. A. Robertson, "Primary unexplained infertility is associated with reduced expression of the T-regulatory cell transcription factor Foxp3 in endometrial tissue," Molecular Human Reproduction, vol. 12, no. 5, pp. 301308, 2006. 
[53] T. Nagamatsu and D. J. Schust, "The contribution of macrophages to normal and pathological pregnancies "'American Journal of Reproductive Immunology, vol. 63, no. 6, pp. 460-471, 2010.

[54] L. Sykes, D. A. MacIntyre, X. J. Yap, T. G. Teoh, and P. R. Bennett, "The Th1:Th2 dichotomy of pregnancy and preterm labour," Mediators of Inflammation, vol. 2012, Article ID 967629, 12 pages, 2012.

[55] B. A. Croy, S. Chantakru, S. Esadeg, A. A. Ashkar, and Q. Wei, "Decidual natural killer cells: Key regulators of placental development (a review)," Journal of Reproductive Immunology, vol. 57, no. 1-2, pp. 151-168, 2002.

[56] J. Hanna, D. Goldman-Wohl, Y. Hamani et al., "Decidual NK cells regulate key developmental processes at the human fetalmaternal interface," Nature Medicine, vol. 12, no. 9, pp. 10651074, 2006.

[57] O. Guzeloglu-Kayisli, U. A. Kayisli, and H. S. Taylor, “The role of growth factors and cytokines during implantation:Endocrine and paracrine interactions," Seminars in Reproductive Medicine, vol. 27, no. 1, pp. 62-79, 2009.

[58] A. M. Sharkey, A. King, D. E. Clark et al., "Localization of leukemia inhibitory factor and its receptor in human placenta throughout pregnancy," Biology of Reproduction, vol. 60, no. 2, pp. 355-364, 1999.

[59] H. Nakamura, M. J. Jasper, M. L. Hull, J. D. Aplin, and S. A. Robertson, "Macrophages regulate expression of $\alpha 1,2$-fucosyltransferase genes in human endometrial epithelial cells," Molecular Human Reproduction, vol. 18, no. 4, Article ID gar070, pp. 204-215, 2012.

[60] M. J. Jasper, A. S. Care, B. Sullivan, W. V. Ingman, J. D. Aplin, and S. A. Robertson, "Macrophage-derived LIF and IL1B regulate alpha(1,2)fucosyltransferase 2 (Fut2) expression in mouse uterine epithelial cells during early pregnancy," Biology of Reproduction, vol. 84, no. 1, pp. 179-188, 2011.

[61] N. Yu, J. Yang, Y. Guo et al., "Intrauterine administration of peripheral blood mononuclear cells (PBMCs) improves endometrial receptivity in mice with embryonic implantation dysfunction," American Journal of Reproductive Immunology, vol. 71, no. 1, pp. 24-33, 2014.

[62] O. Okitsu, M. Kiyokawa, T. Oda, K. Miyake, Y. Sato, and H. Fujiwara, "Intrauterine administration of autologous peripheral blood mononuclear cells increases clinical pregnancy rates in frozen/thawed embryo transfer cycles of patients with repeated implantation failure," Journal of Reproductive Immunology, vol. 92, no. 1-2, pp. 82-87, 2011.

[63] K. K. Niakan, J. Han, R. A. Pedersen, C. Simon, and R. A. R. Pera, "Human pre-implantation embryo development," Development, vol. 139, no. 5, pp. 829-841, 2012.

[64] R. J. Naftalin, J. R. Thiagarajah, K. C. Pedley, V. J. Pocock, and S. R. Milligan, "Progesterone stimulation of fluid absorption by the rat uterine gland," Reproduction, vol. 123, no. 5, pp. 633-638, 2002.

[65] P. Xenopoulos, M. Kang, and A.-K. Hadjantonakis, "Cell lineage allocation within the inner cell mass of the mouse blastocyst," in Mouse Development, J. Z. Kubiak, Ed., pp. 185-202, Springer, Berlin, Germany, 2012.

[66] B. Movaghar and S. Askarian, "Expression of e-cadherin, leukemia inhibitory factor and progesterone receptor in mouse blastocysts after ovarian stimulation," Cell Journal, vol. 14, no. 3 , pp. 225-230, 2012.

[67] H. Tsai, C. Chang, Y. Hsieh, L. Hsu, S. Chang, and H. Lo, "Effect of different concentrations of recombinant leukemia inhibitory factor on different development stage of mouse embryo in vitro," Journal of Assisted Reproduction and Genetics, vol. 17, no. 6, pp. 352-355, 2000.

[68] L. Cheung, H. Leung, and A. Bongso, "Effect of supplementation of leukemia inhibitory factor and epidermal growth factor on murine embryonic development in vitro, implantation, and outcome of offspring," Fertility and Sterility, vol. 80, no. 2, pp. 727-735, 2003.

[69] H.-F. Chen, J. Shew, H. Ho, W. Hsu, and Y. Yang, "Expression of leukemia inhibitory factor and its receptor in preimplantation embryos," Fertility and Sterility, vol. 72, no. 4, pp. 713-719, 1999.

[70] T. Lei, Z. Q. Yang, T. Xia et al., "Stage-specific expression of leukaemia inhibitory factor and its receptor in rabbit preimplantation embryo and uterine epithelium during early pregnancy," Reproduction in Domestic Animals, vol. 39, no. 1, pp. $13-18,2004$.

[71] M. J. T. van Eijk, J. Mandelbaum, J. Salat-Baroux et al., "Expression of leukaemia inhibitory factor receptor subunits LIFR $\beta$ and gp130 in human oocytes and preimplantation embryos," Molecular Human Reproduction, vol. 2, no. 5, pp. 355-360, 1996.

[72] F. Hambiliki, J. Hanrieder, J. Bergquist, J. Hreinsson, A. Stavreus-Evers, and K. Wånggren, "Glycoprotein 130 promotes human blastocyst development in vitro," Fertility and Sterility, vol. 99, no. 6, pp. 1592-1599.e3, 2013.

[73] J. A. Neira, D. Tainturier, M. A. Peña, and J. Martal, "Effect of the association of IGF-I, IGF-II, bFGF, TGF- $\beta 1$, GM-CSF, and LIF on the development of bovine embryos produced in vitro," Theriogenology, vol. 73, no. 5, pp. 595-604, 2010.

[74] P. Fedorcsák and R. Storeng, "Effects of leptin and leukemia inhibitory factor on preimplantation development and STAT3 signaling of mouse embryos in vitro "' Biology of Reproduction, vol. 69, no. 5, pp. 1531-1538, 2003.

[75] K. Leduc, V. Bourassa, É. Asselin, P. Leclerc, J. Lafond, and C. Reyes-Moreno, "Leukemia inhibitory factor regulates differentiation of trophoblastlike BeWo cells through the activation of JAK/STAT and MAPK3/1 MAP kinase-signaling pathways," Biology of Reproduction, vol. 86, no. 2, article 54, 2012.

[76] C. Mo, W. Chearwae, and J. J. Bright, "PPAR $\gamma$ regulates LIFinduced growth and self-renewal of mouse ES cells through Tyk2-Stat3 pathway," Cellular Signalling, vol. 22, no. 3, pp. 495500, 2010.

[77] M. Meseguer, J. D. Aplin, P. Caballero-Campo et al., "Human endometrial mucin $\mathrm{MUCl}$ is up-regulated by progesterone and down-regulated in vitro by the human blastocyst," Biology of Reproduction, vol. 64, no. 2, pp. 590-601, 2001.

[78] B. Refaat, H. Simpson, E. Britton et al., "Why does the fallopian tube fail in ectopic pregnancy? The role of activins, inducible nitric oxide synthase, and MUC1 in ectopic implantation," Fertility and Sterility, vol. 97, no. 5, pp. 1115-1123, 2012.

[79] D. D. Carson, J. Julian, B. A. Lessey, A. Prakobphol, and S. J. Fisher, "MUC1 is a scaffold for selectin ligands in the human uterus," Frontiers in Bioscience, vol. 11, no. 3, pp. 2903-2908, 2006.

[80] R. Nejatbakhsh, M. Kabir-Salmani, E. Dimitriadis et al., "Subcellular localization of L-selectin ligand in the endometrium implies a novel function for pinopodes in endometrial receptivity," Reproductive Biology and Endocrinology, vol. 10, article 46, 2012.

[81] M. Zhao, C. Chang, Z. Liu, L. M. Chen, and Q. Chen, "Treatment with low-dose aspirin increased the level LIF and integrin $\beta 3$ expression in mice during the implantation window," Placenta, vol. 31, no. 12, pp. 1101-1105, 2010. 
[82] M. N. Fukuda and K. Sugihara, "An integrated view of L-selectin and trophinin function in human embryo implantation," Journal of Obstetrics and Gynaecology Research, vol. 34, no. 2, pp. 129-136, 2008.

[83] R.-W. Su, B. Jia, H. Ni et al., "Junctional adhesion molecule 2 mediates the interaction between hatched blastocyst and luminal epithelium: induction by progesterone and LIF," PLoS ONE, vol. 7, no. 4, Article ID e34325, 2012.

[84] G. Raab, K. Kover, B. C. Paria, S. K. Dey, R. M. Ezzell, and M. Klagsbrun, "Mouse preimplantation blastocysts adhere to cells expressing the transmembrane form of heparin-binding EGFlike growth factor," Development, vol. 122, no. 2, pp. 637-645, 1996.

[85] L. Aghajanova, A. Stavreus-Evers, Y. Nikas, O. Hovatta, and B. Landgren, "Coexpression of pinopodes and leukemia inhibitory factor, as well as its receptor, in human endometrium," Fertility and Sterility, vol. 79, supplement 1, pp. 808-814, 2003.

[86] L. Li, B. Xu, Q. Chen, and X. Sun, "Effects of hydrosalpinx on pinopodes, leukaemia inhibitory factor, integrin $\beta 3$ and MUC1 expression in the peri-implantation endometrium," European Journal of Obstetrics Gynecology and Reproductive Biology, vol. 151, no. 2, pp. 171-175, 2010.

[87] B. Xu, X. Sun, L. Li, L. Wu, A. Zhang, and Y. Feng, "Pinopodes, leukemia inhibitory factor, integrin- $\beta 3$, and mucin- 1 expression in the peri-implantation endometrium of women with unexplained recurrent pregnancy loss," Fertility and Sterility, vol. 98, no. 2, pp. 389-395, 2012.

[88] L. N. Kent, T. Konno, and M. J. Soares, "Phosphatidylinositol 3 kinase modulation of trophoblast cell differentiation," $B M C$ Developmental Biology, vol. 10, article 97, 2010.

[89] M. Hemberger, "IFPA Award in Placentology Lecture. Characteristics and significance of trophoblast giant cells," Placenta, vol. 29, pp. 4-9, 2008.

[90] P. Bischof and A. Campana, "Molecular mediators of implantation," Bailliere's Best Practice and Research in Clinical Obstetrics and Gynaecology, vol. 14, no. 5, pp. 801-814, 2000.

[91] S. X. Bai, Y. Wang, L. Qin, Z. J. Xiao, R. Herva, and Y. Piao, "Dynamic expression of matrix metalloproteinases (MMP-2, -9 and -14) and the tissue inhibitors of MMPs (TIMP-1, -2 and -3) at the implantation site during tubal pregnancy," Reproduction, vol. 129, no. 1, pp. 103-113, 2005.

[92] T. G. Poehlmann, J. S. Fitzgerald, A. Meissner et al., "Trophoblast invasion: tuning through LIF, signalling via Stat3," Placenta, vol. 26, pp. S37-S41, 2005.

[93] F. M. Corvinus, J. S. Fitzgerald, K. Friedrich, and U. R. Markert, "Evidence for a correlation between trophoblast invasiveness and STAT3 activity," American Journal of Reproductive Immunology, vol. 50, no. 4, pp. 316-321, 2003.

[94] G. J. Prakash, P. Suman, D. M. M. Prieto, U. R. Markert, and S. K. Gupta, "Leukaemia inhibitory factor mediated proliferation of HTR-8/SVneo trophoblast cells is dependent on activation of extracellular signal-regulated kinase 1/2," Reproduction, Fertility and Development, vol. 23, no. 5, pp. 714-724, 2011.

[95] P. Suman, N. Shembekar, and S. K. Gupta, "Leukemia inhibitory factor increases the invasiveness of trophoblastic cells through integrated increase in the expression of adhesion molecules and pappalysin 1 with a concomitant decrease in the expression of tissue inhibitor of matrix metalloproteinases," Fertility and Sterility, vol. 99, no. 2, pp. 533.e2-542.e2, 2013.

[96] A. Tapia, L. A. Salamonsen, U. Manuelpillai, and E. Dimitriadis, "Leukemia inhibitory factor promotes human first trimester extravillous trophoblast adhesion to extracellular matrix and secretion of tissue inhibitor of metalloproteinases-1 and -2," Human Reproduction, vol. 23, no. 8, pp. 1724-1732, 2008.

[97] S. Gremlich, A. Chanson, F. Urner et al., "LIF and sIL-2R plasma concentrations in IVF patients on the day of embryo transfer: predictive markers of IVF outcome," Journal of Reproductive Immunology, vol. 94, no. 2, pp. 175-182, 2012.

[98] P. R. Brinsden, V. Alam, B. de Moustier, and P. Engrand, "Recombinant human leukemia inhibitory factor does not improve implantation and pregnancy outcomes after assisted reproductive techniques in women with recurrent unexplained implantation failure," Fertility and Sterility, vol. 91, no. 4, pp. 1445-1447, 2009.

[99] J. Terakawa, S. Wakitani, M. Sugiyama et al., "Embryo implantation is blocked by intraperitoneal injection with anti-LIF antibody in mice," Journal of Reproduction and Development, vol. 57, no. 6, pp. 700-707, 2011.

[100] A. R. Lemons and R. K. Naz, "Birth control vaccine targeting leukemia inhibitory factor," Molecular Reproduction and Development, vol. 79, no. 2, pp. 97-106, 2012.

[101] J. Sengupta, P. G. L. Lalitkumar, A. R. Najwa, and D. Ghosh, "Monoclonal anti-leukemia inhibitory factor antibody inhibits blastocyst implantation in the rhesus monkey," Contraception, vol. 74, no. 5, pp. 419-425, 2006.

[102] C. A. White, J. Zhang, L. A. Salamonsen et al., "Blocking LIF action in the uterus by using a PEGylated antagonist prevents implantation: a nonhormonal contraceptive strategy," Proceedings of the National Academy of Sciences of the United States of America, vol. 104, no. 49, pp. 19357-19362, 2007.

[103] E. Menkhorst, J. Zhang, N. A. Sims et al., "Vaginally administered PEGylated LIF Antagonist blocked embryo implantation and eliminated non-target effects on bone in mice," PLOS ONE, vol. 6, no. 5, Article ID e19665, 2011.

[104] L. C. Aschenbach, K. E. Hester, N. C. McCann, J. Zhang, E. Dimitriadis, and D. M. Duffy, "The LIF receptor antagonist PEGLA is effectively delivered to the uterine endometrium and blocks LIF activity in cynomolgus monkeys," Contraception, vol. 87, no. 6, pp. 813-823, 2013.

[105] S. Lalitkumar, N. R. Boggavarapu, J. Menezes et al., "Polyethylene glycated leukemia inhibitory factor antagonist inhibits human blastocyst implantation and triggers apoptosis by downregulating embryonic AKT,' Fertility and Sterility, vol. 100, no. 4, pp. 1160.e2-1169.e2, 2013. 


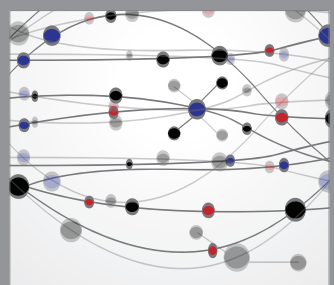

The Scientific World Journal
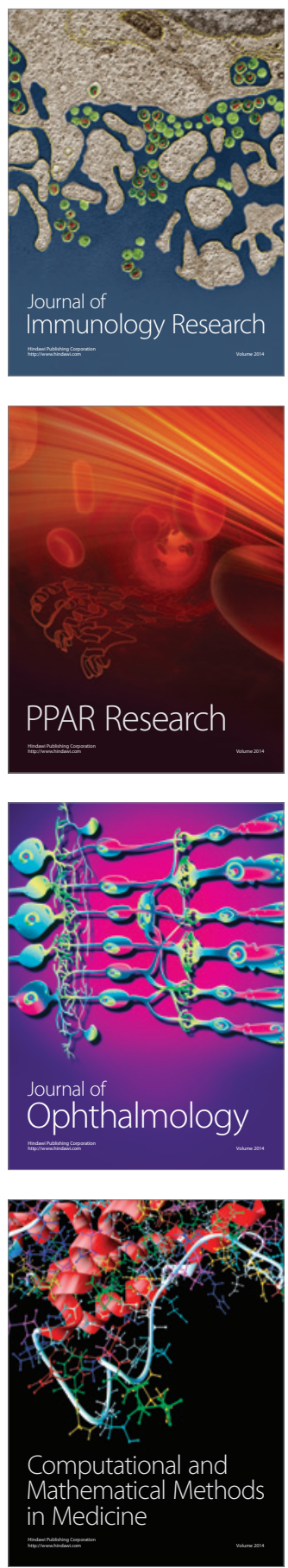

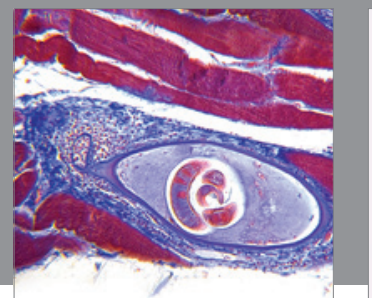

Gastroenterology

Research and Practice
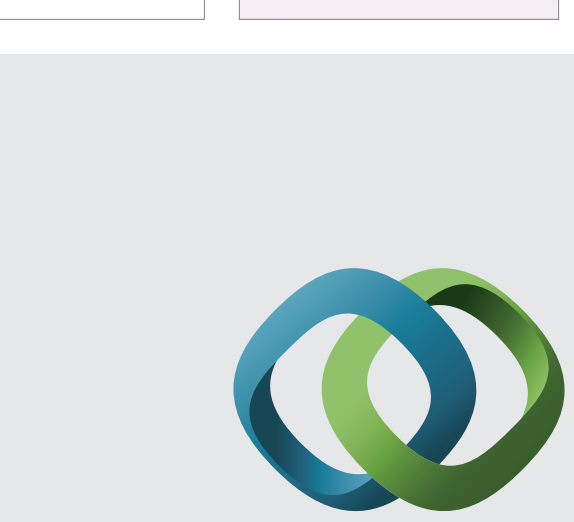

\section{Hindawi}

Submit your manuscripts at

http://www.hindawi.com
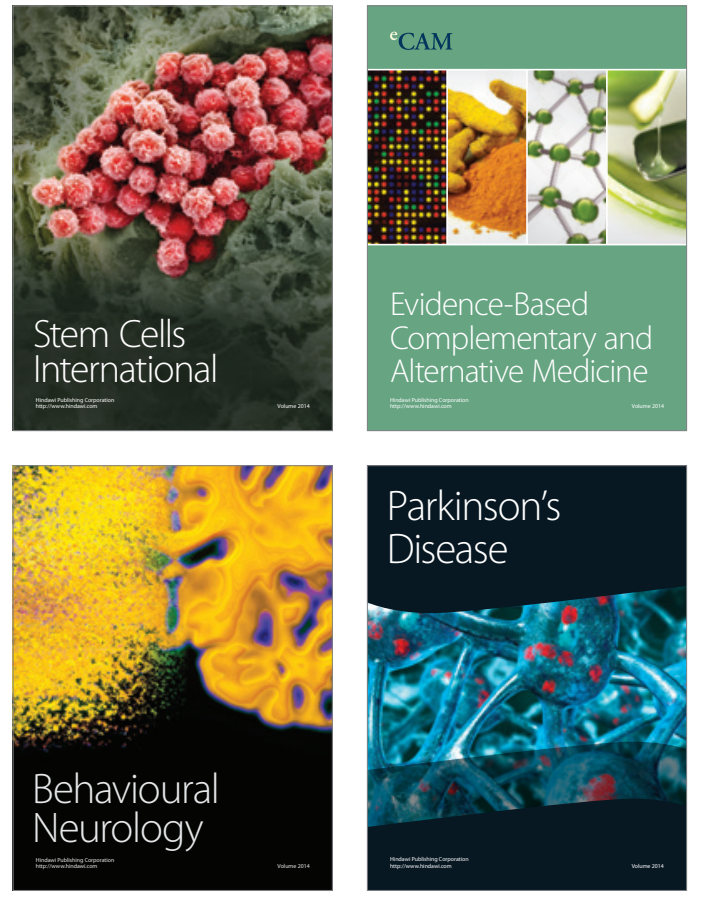
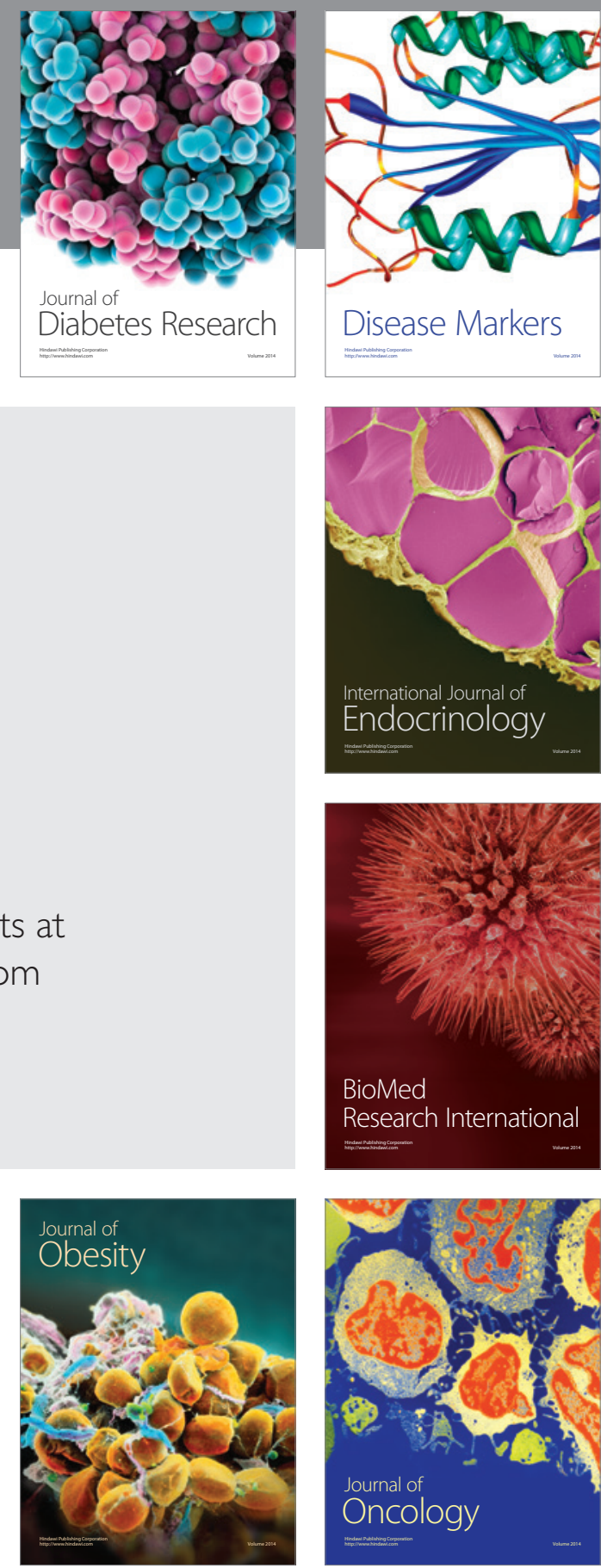

Disease Markers
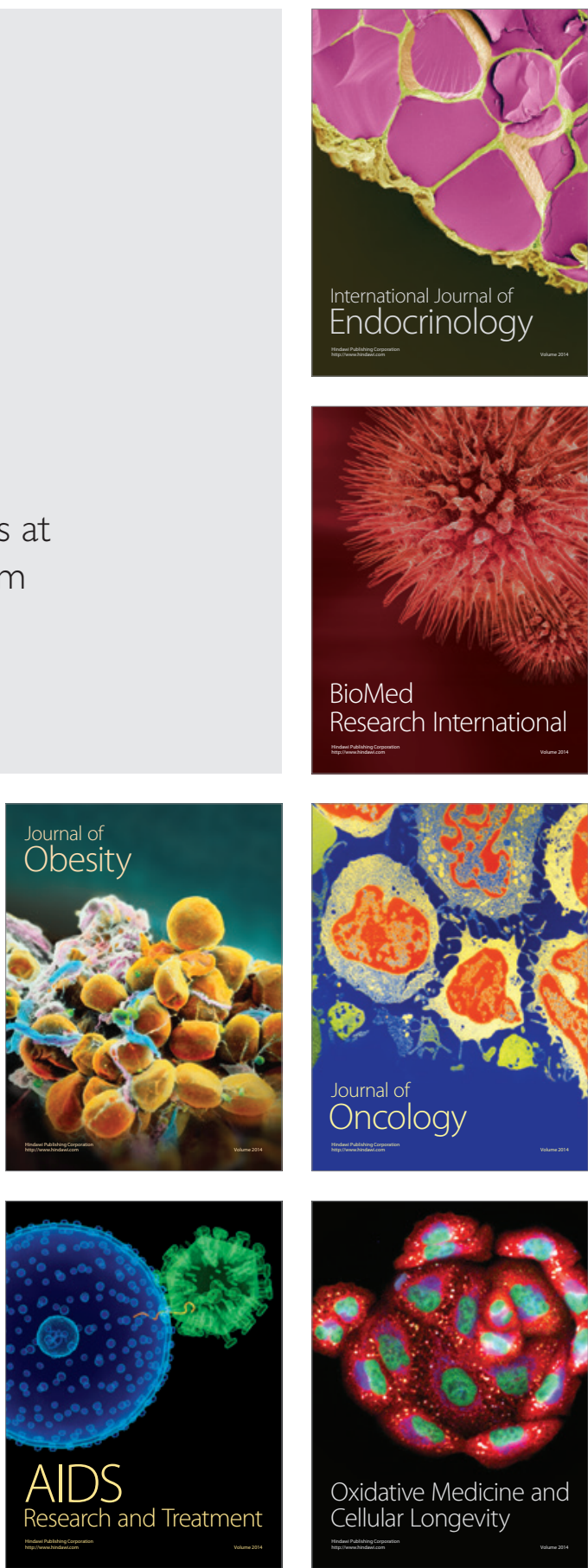